\title{
Biodegradable reduce expenditure bioreactor for augmented sonodynamic therapy via regulating tumor hypoxia and inducing pro-death autophagy
}

Weijuan Zou ${ }^{1,2 \dagger}$, Junnian $\mathrm{Hao}^{1 \dagger}{ }^{\dagger}$, Jianrong Wu ${ }^{1 *}$, Xiaojun Cai ${ }^{1}$, Bing Hu${ }^{1}$, Zhigang Wang ${ }^{2}$ and Yuanyi Zheng ${ }^{1,2,3^{*}}$ (1)

\begin{abstract}
Backgrounds: Sonodynamic therapy (SDT) as an emerging reactive oxygen species (ROS)-mediated antitumor strategy is challenged by the rapid depletion of oxygen, as well as the hypoxic tumor microenvironment. Instead of the presently available coping strategies that amplify the endogenous $\mathrm{O}_{2}$ level, we have proposed a biodegradable $\mathrm{O}_{2}$ economizer to reduce expenditure for augmenting SDT efficacy in the present study.

Results: We successfully fabricated the $\mathrm{O}_{2}$ economizer (HMME@HMONs-3BP-PEG, HHBP) via conjugation of respiration inhibitor 3-bromopyruvate (3BP) with hollow mesoporous organosilica nanoparticles (HMONs), followed by the loading of organic sonosensitizers (hematoporphyrin monomethyl ether; HMME) and further surface modification of poly(ethylene glycol) (PEG). The engineered HHBP features controllable pH/GSH/US-sensitive drug release. The exposed 3BP could effectively inhibit cell respiration for restraining the oxygen consumption, which could alleviate the tumor hypoxia conditions. More interestingly, it could exorbitantly elevate the autophagy level, which in turn induced excessive activation of autophagy for promoting the therapeutic efficacy. As a result, when accompanied with suppressing $\mathrm{O}_{2}$-consumption and triggering pro-death autophagy strategy, the HHBP could achieve the remarkable antitumor activity, which was systematically validated both in vivo and in vitro assays.
\end{abstract}

Conclusions: This work not only provides a reduce expenditure means for enduring SDT, but also represents an inquisitive strategy for tumor treatments by inducing pro-death autophagy.

Keywords: Sonodynamic therapy, Hypoxia regulation, Pro-death autophagy, Hollow mesoporous organosilica, Biodegradable give appropriate credit to the original author(s) and the source, provide a link to the Creative Commons licence, and indicate if changes were made. The images or other third party material in this article are included in the article's Creative Commons licence, unless indicated otherwise in a credit line to the material. If material is not included in the article's Creative Commons licence and your intended use is not permitted by statutory regulation or exceeds the permitted use, you will need to obtain permission directly from the copyright holder. To view a copy of this licence, visit http://creativecommons.org/licenses/by/4.0/. The Creative Commons Public Domain Dedication waiver (http://creativecommons.org/publicdomain/zero/1.0/) applies to the data made available in this article, unless otherwise stated in a credit line to the data. 


\section{Graphical Abstract}

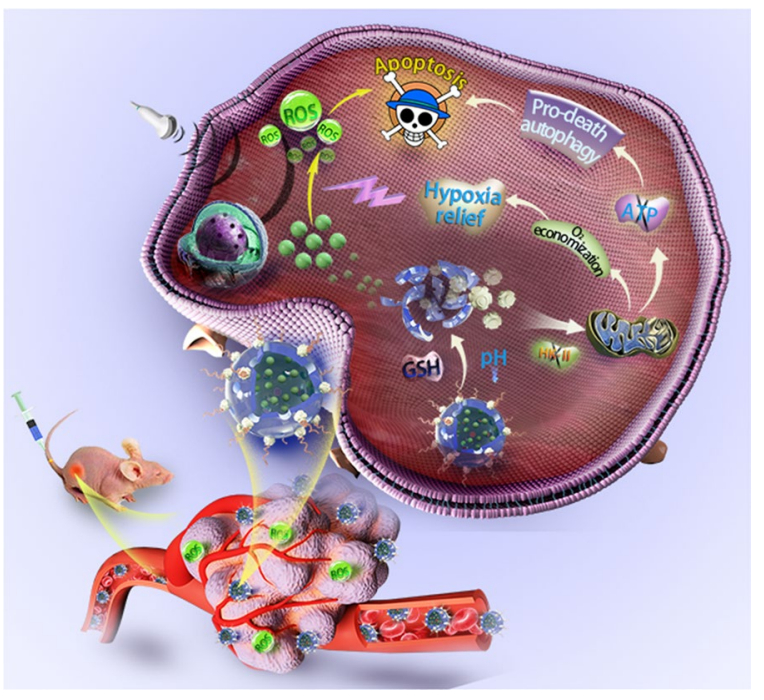

\section{Introduction}

As an emerging cancer-treatment modality, sonodynamic therapy (SDT) employs sonosensitizers that are activated by ultrasound (US) in the presence of oxygen, which then enables the generation of highly toxic reactive oxygen species (ROS) in cancer cells, primarily singlet oxygen $\left({ }^{1} \mathrm{O}_{2}\right)[1-3]$. Due to the deep tissue penetration of the US, SDT offers multiple advantages, such as noninvasiveness and high tissue-penetrating depth relative to that with photodynamic therapy, thereby promising better potential for the treatment of deep-seated tumors [47]. Although preclinical researches on SDT have shown promising results in various cancer cells, some undesirable limitations severely restricting the clinical translation of SDT. For instance, the efficacy of SDT mainly depends on oxygen content inside the solid tumors, while several solid tumors often feature hypoxia triggered by the limited oxygen diffusion and the rapid proliferation of cancer cells [8-11]. However, this situation is further challenged by the fact that hypoxia gets further intensified during the SDT process, triggering SDT resistance. Thus, overcoming tumor hypoxia is the priority among priorities in SDT process. In principle, the hypoxic tumor microenvironment (TME) can be reshaped by improving the exogenous $\mathrm{O}_{2}$ level or suppressing $\mathrm{O}_{2}$ consumption [12-14]. Promoted by the significant advances in nanomedicine, several expectant strategies aimed at modulating the level of oxygen in a solid tumor, such as delivering $\mathrm{O}_{2}$ to the hypoxic regions via oxygen-carrying nanomaterials and the in situ generations of $\mathrm{O}_{2}$ by nanocatalysts/nanozymes-mediated hydrogen peroxide
$\left(\mathrm{H}_{2} \mathrm{O}_{2}\right)$ decomposition, have been exploited [11, 15-20]. Nevertheless, the feasibility of these strategies is severely hindered by the leakage of $\mathrm{O}_{2}$ from the carriers and the insufficient endogenous $\mathrm{H}_{2} \mathrm{O}_{2}[3,21-23]$. Compared to the widespread tactics of improving the oxygen content, reducing the oxygen consumption seems like a promising alternative to relieve tumor hypoxia. It has been proved so far that inhibiting mitochondrial respiration by some oxygen-regulator (e.g., NO, [24] atovaquone [25] and metformin [26]) can effectively oxygenate tumors for improving the therapeutic efficacies. Unfortunately, the nanoplatform design that can modulate hypoxia by reducing $\mathrm{O}_{2}$ consumption is still necessary, albeit largely unexploited, especially on oxygen-dependent therapeutic modalities.

In addition to induce cell apoptosis, the potential benefit of the production of ${ }^{1} \mathrm{O}_{2}$ during the SDT process can induce autophagy [27-29]. Autophagy is a cellular degradation process that refers to the degradation of phagocytes cytoplasmic proteins or organelles through the formation of autophagy lysosomes [30, 31], which commonly considered as a self-protection process aimed at achieving the renewal of some organelles and the metabolism and energy of the cell itself [32]. However, autophagy is a "double-edged sword". When it exceeds a certain threshold, exorbitant and abnormal autophagy can promote cell death under specific circumstances [33, 34]. It can thus be further hypothesized that the activation of pro-death autophagy can be a potential strategy to improve the efficacy of cancer treatment. Considering that autophagy induced by ROS is pro-survival, the 
construction of nanosystems with both the sonosensitizer activity and the capability of regulating autophagy from self-protection to pro-death is expected to achieve augmented SDT.

Considering the above discussed assumptions, we have proposed a bioreactor with reduced expenditure for augmenting SDT efficacy through the regulation of tumor hypoxia and induction of pro-death autophagy. This bioreactor was constructed by decorating respiration inhibitor 3-bromopyruvate (3BP) onto the surface of disulfide bonds-hybridized hollow mesoporous organosilica nanoparticles (HMONs) via an amide linker. Organic sonosensitizers (hematoporphyrin monomethyl ether, HMME) were further loaded into the mesopores and the hollow interior of HMONs, followed by modification with poly (ethylene glycol) (PEG) (HMME@ HMONs-3BP-PEG, designated as HHBP) to enhance their biocompatibility (Fig. 1). The presently engineered HHBP have featured intrinsic TME-responsive biodegradability that function as a nanosonosensitizer to produce ${ }^{1} \mathrm{O}_{2}$ under the US irradiation. The delivered 3BP significantly reduces the intracellular oxygen consumption rate by perturbing the respiration process, thus relieving tumor hypoxia for further augmenting the production of ${ }^{1} \mathrm{O}_{2}$. Specifically, 3BP as a hexokinase type II (HK-II) inhibitor, can block the first stage of respiration to inhibit both anaerobic and aerobic respiration. Correspondingly, glycolysis was inhibited, which caused the reduction of intracellular production of lactate and adenosine triphosphate (ATP). Therefore, 3BP is expected to shut down the nutrient supply for inhibiting cellular respiration for the rapid depletion of ATP and the simultaneous induction of autophagy $[35,36]$. The combination of ROS production and ATP supply restriction results in the changeover of autophagy from self-protection to pro-death, which in turn promotes cell apoptosis. As a result, enhanced SDT therapeutic outcome could be acquired, which was systematically investigated both in vitro at the cellular level and in vivo on $4 \mathrm{~T} 1$ tumor-bearing mice. These findings demonstrate the feasibility of the present augmented SDT strategy based on the 3BP and HMME co-delivered systems. Our work provides an expenditure reduction strategy for facilitating ROS production, while simultaneously offering a rationale for the combination of respiration inhibition and pro-death autophagy therapy as a therapeutic strategy against cancer.

\section{Methods \\ Synthesis of hollow mesoporous organosilica nanoparticles (HMONs)}

The HMONs were prepared as described in our previous work [37, 38]. Briefly, cetyltrimethylammonium chloride

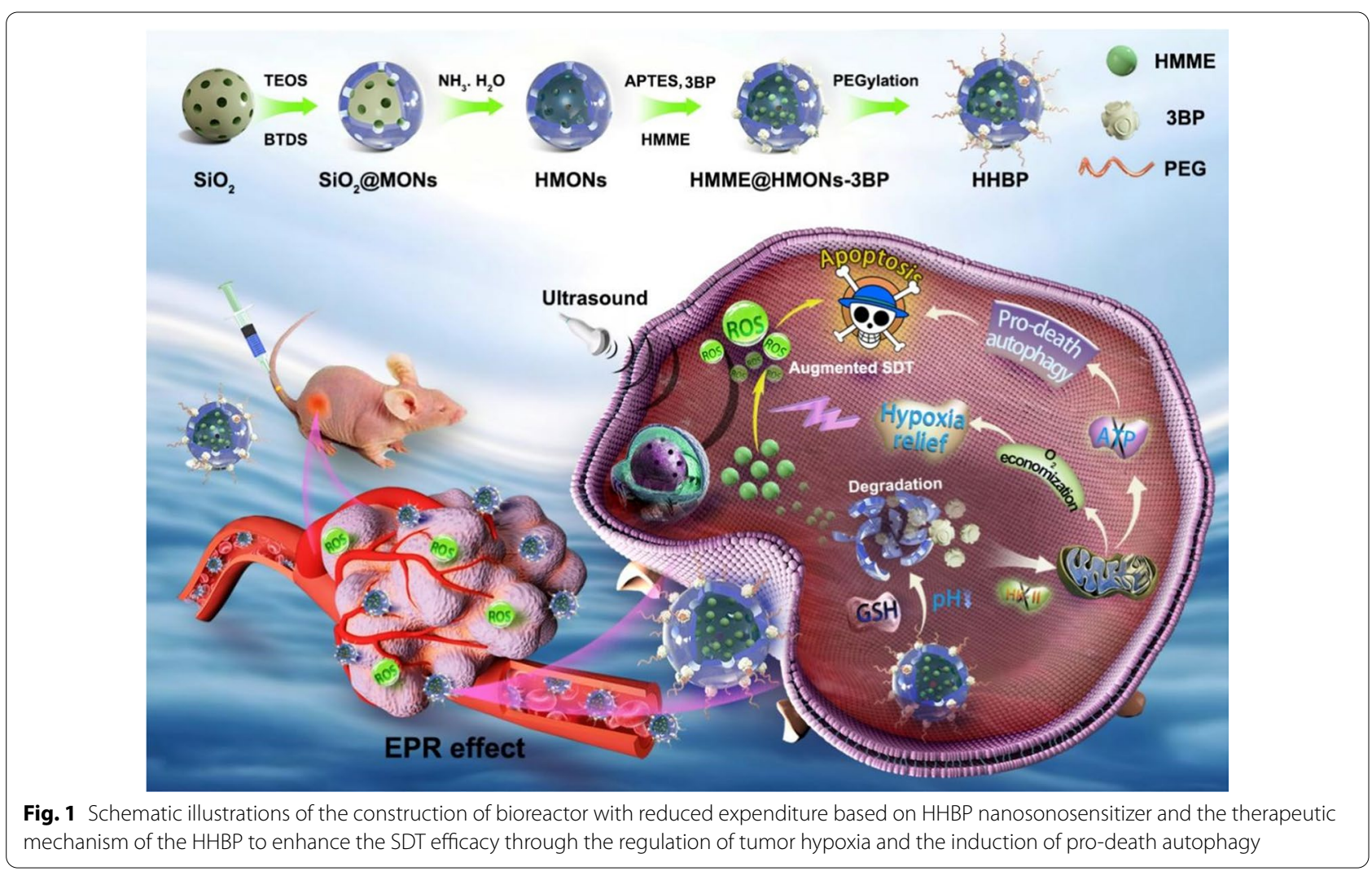


(CTAC, $10 \mathrm{~g}$ ) was dissolved in deionized water $(100 \mathrm{~mL})$, to which triethanolamine $(8 \mathrm{~g})$ solution was added. The resultant mixture was heated and stirred in an oil bath to $80{ }^{\circ} \mathrm{C}$ and tetraethyl orthosilicate (TEOS, $0.5 \mathrm{~mL}$ ) was added dropwise for $1 \mathrm{~h}$. Then, a mixture of TEOS (1 $\mathrm{mL}$ ) and bis(3-triethoxysilylpropyl) disulfide (BTDS, 0.6 $\mathrm{mL}$ ) was immediately added to continue the reaction for $4 \mathrm{~h}$ to form the core/shell structure. The products were washed thrice with a mixture of ethanol $(50 \mathrm{~mL})$ and hydrochloric acid $(4 \mathrm{~mL})$ in a reflux unit at $80{ }^{\circ} \mathrm{C}$. Then, the products were dispersed into deionized water $(20$ $\mathrm{mL}$ ), followed by the addition of ammonium hydroxide $(3.5 \mathrm{~mL})$ and incubation for another $3 \mathrm{~h}$ at $98{ }^{\circ} \mathrm{C}$. The final HMON products were obtained through centrifugation, washing and dried under vacuum.

\section{Synthesis of HHBP nanoparticles}

HMONs $(200 \mathrm{mg})$ were dispersed in the methanol solution and functionalized with 3-aminopropyltriethoxysilane (APTES, $1 \mathrm{~mL}$ ) for $16 \mathrm{~h}$ at $120{ }^{\circ} \mathrm{C}$. Then, the precipitate was collected by centrifugation, washed with ethanol several times, and finally dried to obtain the HMONs- $\mathrm{NH}_{2}$ nanoparticles. Next, the 3BP was anchored onto the surface of $\mathrm{HMONs}-\mathrm{NH}_{2}$ via the amidation reaction. Briefly, $\mathrm{HMONs}-\mathrm{NH}_{2}(80 \mathrm{mg})$ nanoparticles were dispersed in $\mathrm{DMSO} /$ deionized water mixture solution, containing EDC (12.0 mg) and NHS (7 mg, 0.12 $\mathrm{mmoL})$. The reaction system was stirred for $6 \mathrm{~h}$ at room temperature, to which 3BP (10 mg) was slowly added, followed by vigorous stirring for another $12 \mathrm{~h}$. The product (HMONs-3BP) was obtained via centrifugation and purified with ethanol.

For hematoporphyrin monomethyl ether (HMME) loading, HMONs-3BP was dispersed in the HMME methanol solution at different mass ratios $(4: 1 ; 2: 1 ; 1: 1$; $2: 3 ; 2: 4)$ and stirred at room temperature for $24 \mathrm{~h}$. After centrifugation and purification with methanol several times, the HMME-loaded formulations (designated as HMME@HMONs-3BP) were obtained. Meanwhile, the collected supernatant was evaluated for its HMME-loading capacity by UV-vis analysis. Finally, the PEGylated nanoparticles (HHBP) were added to the mPEG-COOH in the HMME@HMONs-3BP dispersion and stirred for $12 \mathrm{~h}$. The final product obtained was washed and re-dispersed in PBS for further use.

\section{Qualitative and quantitative ${ }^{1} \mathrm{O}_{2}$ detection}

The generation of ${ }^{1} \mathrm{O}_{2}$ was determined by electron spin resonance (ESR; Brooke A300, Germany) with the trapping agent 2,2,6,6-tetramethylpiperidine (TEMP). Typically, HHBP $(150 \mu \mathrm{g} / \mathrm{mL})$ was exposed to US irradiation $\left(1.0 \mathrm{~W} / \mathrm{cm}^{2}, 1.0 \mathrm{MHz}, 50 \%\right.$ duty circle) for $1 \mathrm{~min}$ in a mixture containing TEMP $(100 \mathrm{mM})$, and the mixture was immediately subjected to ESR spectroscopy. As control groups, PBS with and without US irradiation and HHBP without US irradiation were also tested.

To quantitatively evaluate the production of ${ }^{1} \mathrm{O}_{2}, \mathrm{HHBP}$ was dispersed in DMF $(150 \mu \mathrm{g} / \mathrm{mL}, 2.96 \mathrm{~mL})$, followed by the addition of 1,3-diphenylisobenzofuran (DPBF, $8 \mathrm{mM}$, $40 \mu \mathrm{L})$. Then, the mixture was treated with US irradiation $\left(1.0 \mathrm{~W} / \mathrm{cm}^{2}, 1.0 \mathrm{MHz}, 50 \%\right.$ duty circle) for $1 \mathrm{~min}$, and the absorbance intensity was recorded by UV-vis spectrophotometer at the wavelength of $398 \mathrm{~nm}$.

\section{In vitro $\mathrm{pH} / \mathrm{GSH} / \mathrm{US}$-responsive HMME release}

First, the biodegradation behavior of the HHBP nanoparticles in the simulated body fluid (SBF) containing GSH at different concentrations $(0,5$, and $10 \mathrm{mM})$ was evaluated by transmission electron microscopy (TEM) observation. The release behavior of HMME was measured by using a dialysis method through a UV-Vis spectrophotometer. Typically, the HHBP dispersion $(1 \mathrm{mg} / \mathrm{mL}$ HMME equivalent, $0.5 \mathrm{~mL}$ DMSO, and $4.5 \mathrm{~mL}$ buffer solution) was placed in a dialysis bag under different incubationcondition groups: (1) PBS, $\mathrm{pH} 7.4$; (2) ABS, $\mathrm{pH} 5.5$; (3) PBS, pH 7.4, GSH (10 mM); and (4) ABS, pH 5.5, GSH $(10 \mathrm{mM})$ at $37^{\circ} \mathrm{C}$ under constant stirring. Meanwhile, 0.5 $\mathrm{mL}$ of the releasing solution outside the dialysis bag was collected for UV-vis analysis at a $398 \mathrm{~nm}$ wavelength to determine the amount of released HMME. In addition, the US-triggered release behavior was assessed under the same condition of group 3 and 4, and then exposed to US irradiation $\left(1.0 \mathrm{~W} / \mathrm{cm}^{2}, 1.0 \mathrm{MHz}, 50 \%\right.$ duty circle) over $1 \mathrm{~min}$ at $1,4,8$, and $16 \mathrm{~h}$ of incubation.

\section{In vitro ROS production at cellular level}

2,7'-dichlorodihydrofluorescein diacetate (DCFH-DA) was served as a probe to detect intracellular ROS generation. Briefly, 4T1 cells were incubated into 12-well plates at the density of $1 \times 10^{5}$ cells overnight. Subsequently, the cell medium was replaced and the cells were treated with different formulations including PBS, HMME@HMONsPEG, and HHBP for another $4 \mathrm{~h}$. Then, the cells were further exposed to US irradiation $\left(1.0 \mathrm{~W} / \mathrm{cm}^{2}, 1.0 \mathrm{MHz}\right.$, $50 \%$ duty circle, $1 \mathrm{~min}$ ) and incubated with DCFH-DA (1:1000 dilution) for $30 \mathrm{~min}$ according to the manufacturer instructions. The treated cells were then washed thrice with PBS and imaged by fluorescence microscopy. Finally, the cells were collected and subjected to quantitative analysis by measuring the fluorescence intensity by flow cytometry (FACS-Calibur; BD Biosciences).

\section{In vitro nanotherapeutic performance of HHBP}

4T1 cells were seeded into 96-well plates at the density of $1 \times 10^{4}$ cells and treated with PBS, HMME@ 
HMONs-PEG, 3BP-HMONs-PEG or HHBP for $4 \mathrm{~h}$. Then, a part of the cells was further exposed to US irradiation $\left(1.0 \mathrm{~W} / \mathrm{cm}^{2}, 1.0 \mathrm{MHz}, 50 \%\right.$ duty circle, $\left.1 \mathrm{~min}\right)$. After incubation for a total time of $12 \mathrm{~h}$, the cells were washed thrice with PBS and enumerated for cell viability by the CCK- 8 assay. Correspondingly, all treated cells were digested with trypsin and stained by using the CalceinAM/PI kit, according to the manufacturer's instructions. After incubation for $15 \mathrm{~min}$, the cells were visualized under a fluorescence microscope. Next, the cell apoptosis detection assay was performed to further investigate the cell death mechanism. Briefly, 4T1 cells were seeded into 6-well plates for $24 \mathrm{~h}$ and treated as for the CCK- 8 assay. Then, the cells were washed with PBS and collected by centrifugation, followed by treatment with the Annexin V-FITC apoptosis detection kit and quantification by flow cytometer.

\section{Detection of HIF-1 $a$ and HK-II by Western blotting}

The expression of HK-II and hypoxia inducible factor- $1 \alpha$ (HIF- $\alpha)$ in cells was evaluated by Western blotting. The BCA protein concentration determination kit was used to measure the protein concentration, as per the specific method recommended by the manufacturer. Briefly, the $4 \mathrm{~T} 1$ cells were inoculated into 6 -well plates $\left(2 \times 10^{5}\right.$ cells/ well) and incubated with PBS, HMME@HMONs-PEG $(100 \mu \mathrm{g} / \mathrm{mL})$, or HHBP $(100 \mu \mathrm{g} / \mathrm{mL})$ for $4 \mathrm{~h}$. Then, a part of the cells was further exposed to US irradiation $(1.0 \mathrm{~W} /$ $\mathrm{cm}^{2}, 1.0 \mathrm{MHz}, 50 \%$ duty circle, $1 \mathrm{~min}$ ). After incubation for a total time of $6 \mathrm{~h}$, a protease inhibitor was added to the cell lysates to extract the proteins, which were then separated by $10 \%$ sodium dodecyl sulfate-polyacrylamide gel electrophoresis and then transferred onto the polyvinylidene fluoride membrane (Millipore, IPVH00010). The membrane was decolorized and sealed with 5\% skim milk (0.5\%TBST) on a shaker for $1 \mathrm{~h}$. The primary antibodies HIF-1 $\alpha$ (1:2000 dilution, ab16066; Abcam,) and HK- II (1:5000 dilution, ab227198; Abcam) were diluted and incubated with the membrane overnight at $4{ }^{\circ} \mathrm{C}$, followed by washing with TBST thrice and hybridizing with the relevant secondary antibody for $1 \mathrm{~h}$. Finally, the film was observed by chemiluminescence. The protein expression and the band strength were quantified using the Image-Pro Plus 6.0 software. $\beta$-actin served as the loading control. Each treatment included three parallel samples, and the data were expressed as mean \pm standard deviation $(\mathrm{n}=3)$.

\section{Animals and tumor model}

Healthy female BALB/c nude mice (age: 4-6 weeks) were provided by the Animal Center of Shanghai Sixth People's Hospital. All animal experiments were conducted as per the protocols approved by the Institutional Animal Care and Use Committee (IACUC) of Shanghai Jiao Tong University Affiliated Sixth People's Hospital (Animal Welfare Ethics acceptance number No: DWLL2020-0582). The 4T1 cell subcutaneous tumor model was established by subcutaneously injecting $100 \mu \mathrm{L}$ of $2 \times 10^{6}$ cells suspension into the female nude mice. The bodyweight of the mice was monitored and the tumor volume $(\mathrm{V})$ was calculated using the following equation: $\mathrm{V}=0.5 \mathrm{~L} \times \mathrm{W}^{2}$. Where, $\mathrm{L}$ and $\mathrm{W}$ represent the length and width of the tumor, respectively.

\section{In vivo antitumor assays}

When the tumor size reached $150 \mathrm{~mm}^{3}$, the mice were randomly divided into 5 groups $(\mathrm{n}=6$ mice in each group), as follows: (I) PBS, (II) HMME@HMONs-PEG, (III) HHBP, (IV) HMME@HMONs-PEG + US, and (V) HHBP + US. The mice were intravenously injected with $100 \mu \mathrm{L}$ of HMME@HMONs-PEG (HMME: $8 \mathrm{mg} / \mathrm{kg}$ ) or HHBP (HMME: $8 \mathrm{mg} / \mathrm{kg}$, 3BP: $3.4 \mathrm{mg} / \mathrm{kg}$ ), respectively. The parameters of US irradiation were set as follows: $1.5 \mathrm{~W} / \mathrm{cm}^{2}, 1.0 \mathrm{MHz}, 50 \%$ duty circle for $5 \mathrm{~min}$. The mice were administrated with the drugs and irradiated on days 1 and 4 . After $12 \mathrm{~h}$ of treatments, tumor blocks of mice were removed and frozen at $-20{ }^{\circ} \mathrm{C}$ for DHE staining to detect ROS. HIF- $1 \alpha$ staining was performed to measure the extent of tumor hypoxia, and LC3 staining was conducted to detect the autophagy level. After treatments, the tumor-bearing nude mice were sacrificed and their tumor tissues were collected for H\&E, Ki67, and TUNEL staining. In addition, the major issues were dissected to prepare paraffin sections for further H\&E staining.

\section{Statistical analysis}

Data were expressed as the mean or mean \pm standard deviation. The statistical significance was determined by Student's $t$-test: ns: $\mathrm{P}>0.05,{ }^{*} \mathrm{P}<0.05$, ${ }^{* * *} \mathrm{P}<0.01$, ${ }^{* * * *} \mathrm{P}$ $<0.001$. All statistical analyses were conducted using the SPSS software.

\section{Results and discussion \\ Design, fabrication, and characterization of HHBP nanosonosensitizers}

The procedure for the fabrication of HHBP nanosonosensitizer and the corresponding therapeutic mechanism is illustrated in Fig. 1. To endow the nanosonosensitizer with TME-responsive biodegradability, we introduced disulfide bond components into the framework of mesoporous silica to prepare organic-inorganic hybrid HMONs for the delivery of both 3BP and HMME. Typically, HMONs were prepared by employing an "ammonia-assisted selective etching" approach reported [38-40]. Initially, the monodispersed $\mathrm{SiO}_{2} @ \mathrm{MONs}$ nanoparticles were synthesized through the co-hydrolysis of 
TEOS and BTDS by employing CTAC as the structuraldirecting agent $[41,42]$. As visualized by TEM observation, the as-synthesized $\mathrm{SiO}_{2} @ M O N s$ exhibited a core/ shell structure (Additional file 1: Fig. S1). After etching in an ammonia solution, the $\mathrm{SiO}_{2}$ core was removed and the hollow HMONs of approximately $85 \mathrm{~nm}$ size were obtained (Fig. 2a). Elemental mapping images showed the presence of sulfur with the coexistence of silicon, carbon, and oxygen in HMONs (Fig. 2b-f), suggesting the formation of disulfide bond hybrid silsesquioxane framework within HMONs, as further proved by energy-dispersive spectrometry (EDS) analyses (Additional file 1: Fig. S2). Meanwhile, the characteristic $\mathrm{C}$ signals in ${ }^{13} \mathrm{C}$ cross-polarization solid-state NMR spectra and silicon resonances in the ${ }^{29} \mathrm{Si}$ magic-angle spinning confirmed the successful hybridization of disulfide bond into the framework of HMONs (Additional file 1: Fig. S3). The amino-functionalized HMONs ( $\left.\mathrm{HMONs}-\mathrm{NH}_{2}\right)$ were prepared and then covalently modified with $3 \mathrm{BP}$ through an amide reaction between the carboxyl groups of $3 \mathrm{BP}$ and the amino groups of $\mathrm{HMONs}-\mathrm{NH}_{2}$ (HMONs3BP). The presence of peaks of the amide group at 1623 $(\mathrm{C}=\mathrm{O}$ stretching vibration) and $3278 / \mathrm{cm}(\mathrm{N}-\mathrm{H}$ stretching vibration [43]) in the Fourier-transformed infrared (FT-IR) spectrum demonstrated the successful formation of HMONs-3BP (Additional file 1: Fig. S4). According to the $\mathrm{N}_{2}$ absorption-desorption isotherms, the BET surface area of HMONs decreased from $481 \mathrm{~m}^{2} / \mathrm{g}$ to 301 $\mathrm{m}^{2} / \mathrm{g}$ after the incorporation of 3BP (Additional file 1: Fig. S5a), while the pore size decreased from $4.4 \mathrm{~nm}$ to

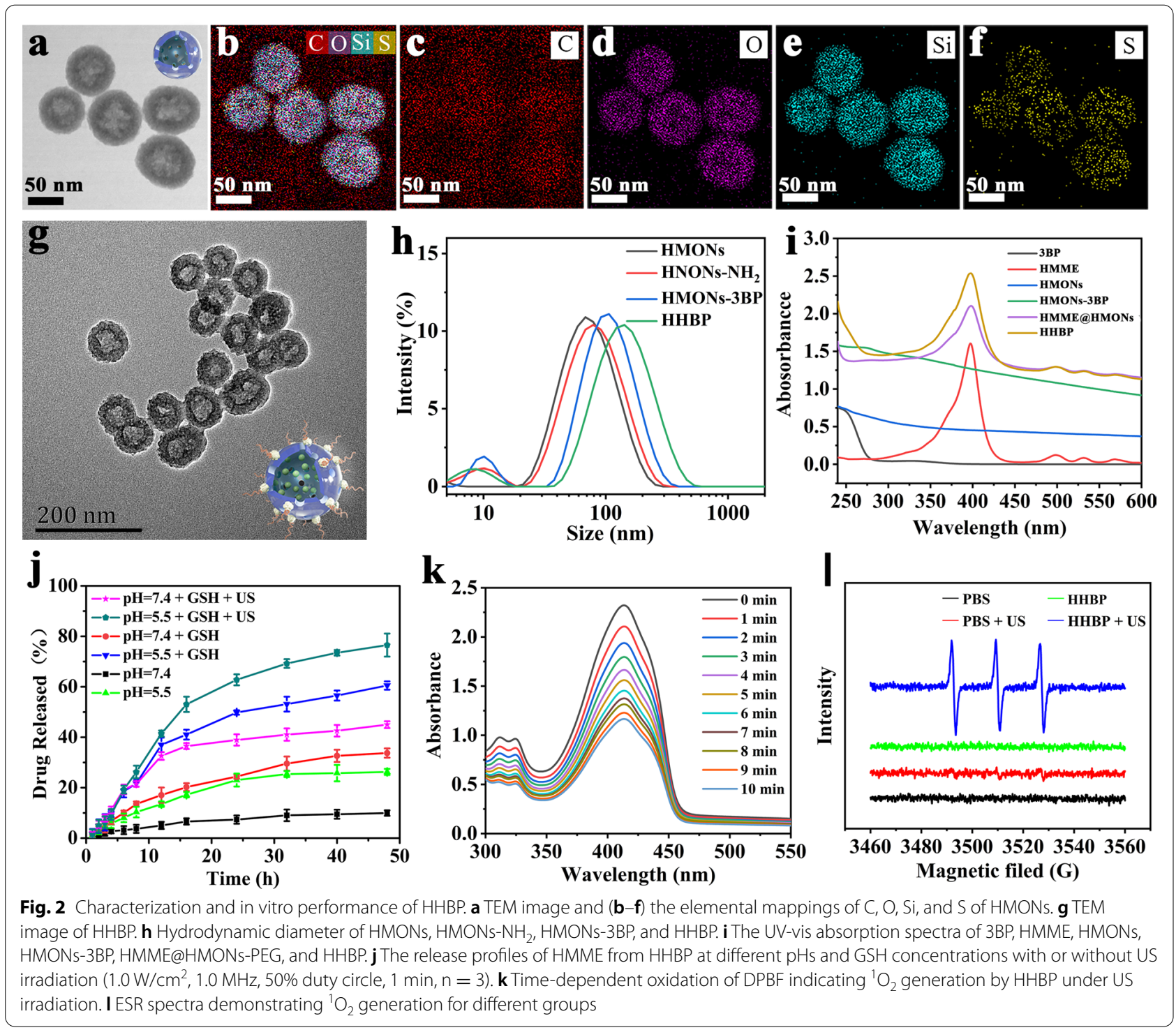


$3.8 \mathrm{~nm}$ approximately (Additional file 1: Fig. S5b). Even so, these characterization results above evidently confirmed the porosity and hollow feature of the as-prepared HMONs-3BP, which is remained highly suitable for the encapsulation of hydrophobic molecules. Thus, organic sonosensitizers HMME was next loaded into the hollow cavity of the HMONs-3BP (designated as HMME@HMONs-3BP). The nanosonosensitizer was further PEGylated through noncovalent interactions for improving the stability (yielding HHBP). TEM observations revealed that the surface engineering and drug loading exhibited a negligible change in morphology (Fig. 2g), while the hydrodynamic diameter of HHBP was increased to $142 \mathrm{~nm}$ (Fig. 2h), which is slightly larger than that of HMONs-3BP. After loading of HMME and PEGylation, the BET surface area was decreased to $206 \mathrm{~m}^{2} / \mathrm{g}$ and the mesopore size was also dramatically reduced to approximately $2.4 \mathrm{~nm}$ (Additional file 1: Fig. S5). Moreover, the serial changes in the zeta potential further reconfirmed the desirable synthesis at each step (Additional file 1: Fig. S6). Furthermore, UV-vis spectra of HHBP depicted the HMME characteristic absorption peaks at $398 \mathrm{~nm}$ (Fig. 2i). The loading capacity of HMME was found to be approximately 38\% (HMME: HMONs$3 \mathrm{BP}, \mathrm{w} / \mathrm{w}$ ) when the HMONs-3BP/ HMME feeding ratio was 0.5 (Additional file 1: Fig. S7). These results suggested that HMONs could be efficiently loaded with HMME and modified with 3BP followed by coating with PEG on the surface. Moreover, HHBP has a good colloid stability and disperse well in PBS, saline and DMEM medium without obvious aggregation, even standing for 7 days. Correspondingly, the absorbance of HHBP at 300-500 nm did not change significantly (Additional file 1: Fig. S8).

As the disulfide-bridged silsesquioxane framework of HMONs can be cleaved in the reductive TME [44, 45], the biodegradation behavior of HHBP was evaluated in the SBF (GSH, $5 \mathrm{mM}$ or $10 \mathrm{mM}$ ). Based on the TEM images, HHBP in SBF without GSH showed a certain extent of albeit inconspicuous biodegradation during 1 week of immersion in SBF. In contrast, HHBP was inclined to be gradually degraded in the SBF solution containing $5 \mathrm{mM} \mathrm{GSH}$, demonstrating a time-dependent biodegradable behavior (Additional file 1: Fig. S9). Especially, the biodegradation rate was significantly quickened and the nanoparticles were found to be entirely biodegraded in the SBF solution containing GSH $(10 \mathrm{mM})$ for $7 \mathrm{~d}$. These results indicated the GSH-responsive biodegradability of the HHBP nanosonosensitizers.

\section{In vitro HMME release and the SDT effect of HHBP}

Considering the unique GSH-sensitive biodegradation behavior of HHBP, we further evaluated their HMME releasing performance and behavior under different GSH concentrations and $\mathrm{pH}$ values (Fig. 2j). As expected, the HMME release behavior from HHBP was highly dependent on the $\mathrm{pH}$ values as well as the GSH concentrations. The percentage of HMME released after $24 \mathrm{~h}$ was found to be $<10 \%$ at $\mathrm{pH} 7.4$ in the absence of $\mathrm{GSH}$, indicating the high stability of HHBP under physiological conditions. In contrast, the amount of released HMME dramatically increased to $26.2 \%$ at $\mathrm{pH} 5.5$, which can be attributed to the electrostatic interaction of HMME with the HMONs was reduced at low $\mathrm{pH}$, thus leading to the HMME release [46]. Notably, the drugreleasing percentage sharply increased to $\approx 33.7 \%$ and $\approx 60.5 \%$ under the respective GSH concentrations of 10 $\mathrm{mM}$ at $\mathrm{pH} 7.4$ and 5.5. This finding can be attributed to the gradual biodegradation of the HMONs framework caused by GSH-induced cleavage of the disulfide bond. Interestingly, the release rates were further enhanced and approximately 76.5\% HMME release was observed after $24 \mathrm{~h}$ upon exposure to US irradiation, which may be attributable to the dissociation of HMME from HMONs caused by the mechanical/cavitation effects of US [2, 47, 48]. Taking into consideration that the tumor environment is distinguished by mild acidic and high GSH concentration, the $\mathrm{pH} / \mathrm{GSH} / \mathrm{US}$ tri-stimuli-responsive HHBP were expected to deliver hydrophobic sonosensitizers for substantially enhanced SDT efficacy.

Subsequently, the SDT performance of HHBP was evaluated based on the presence of HMME, with DPBF as the ${ }^{1} \mathrm{O}_{2}$ probe to determine the ROS production under US irradiation (Fig. 2k). After irradiation with the US, the production of ${ }^{1} \mathrm{O}_{2}$ was further improved, as evidenced by the attenuated UV absorption peak of DPBF at 398 $\mathrm{nm}$ when the US irradiation time increased. In addition, ESR with the spin traps of TEMP were also acquired. According to the ESR spectra (Fig. 2l), the strong ${ }^{1} \mathrm{O}_{2}$ signal $(1: 1: 1)$ was detected in the HHBP + US group, while no obvious ESR signal was observed in the HHBP-alone group and the PBS group, despite irradiation with US $\left(1.0 \mathrm{~W} / \mathrm{cm}^{2}, 1.0 \mathrm{MHz}, 50 \%\right.$ duty circle, $\left.1 \mathrm{~min}\right)$. The above results indicated that HHBP could be an ideal nanosonosensitizer for US-triggered ROS generation.

\section{Intracellularuptake and hypoxia alleviation of HHBP}

Inspired by the aforementioned results, we next evaluated the cellular uptake and $\mathrm{O}_{2}$-consumption reduction ability of HHBP in 4T1 cells. Initially, the in vitro cytotoxicity of HMONs-PEG was determined by using the CCK-8 assay. HMONs-PEG showed no significant cytotoxicity to various cancer cells in vitro (Fig. 3a), including HUVEC, 4T1, A375, and A549 cells, even at high concentrations of $250 \mu \mathrm{g} / \mathrm{mL}$, thereby demonstrating the relatively good biocompatibility of HMONs-PEG. In order to evaluate the intracellular endocytosis behavior, the 

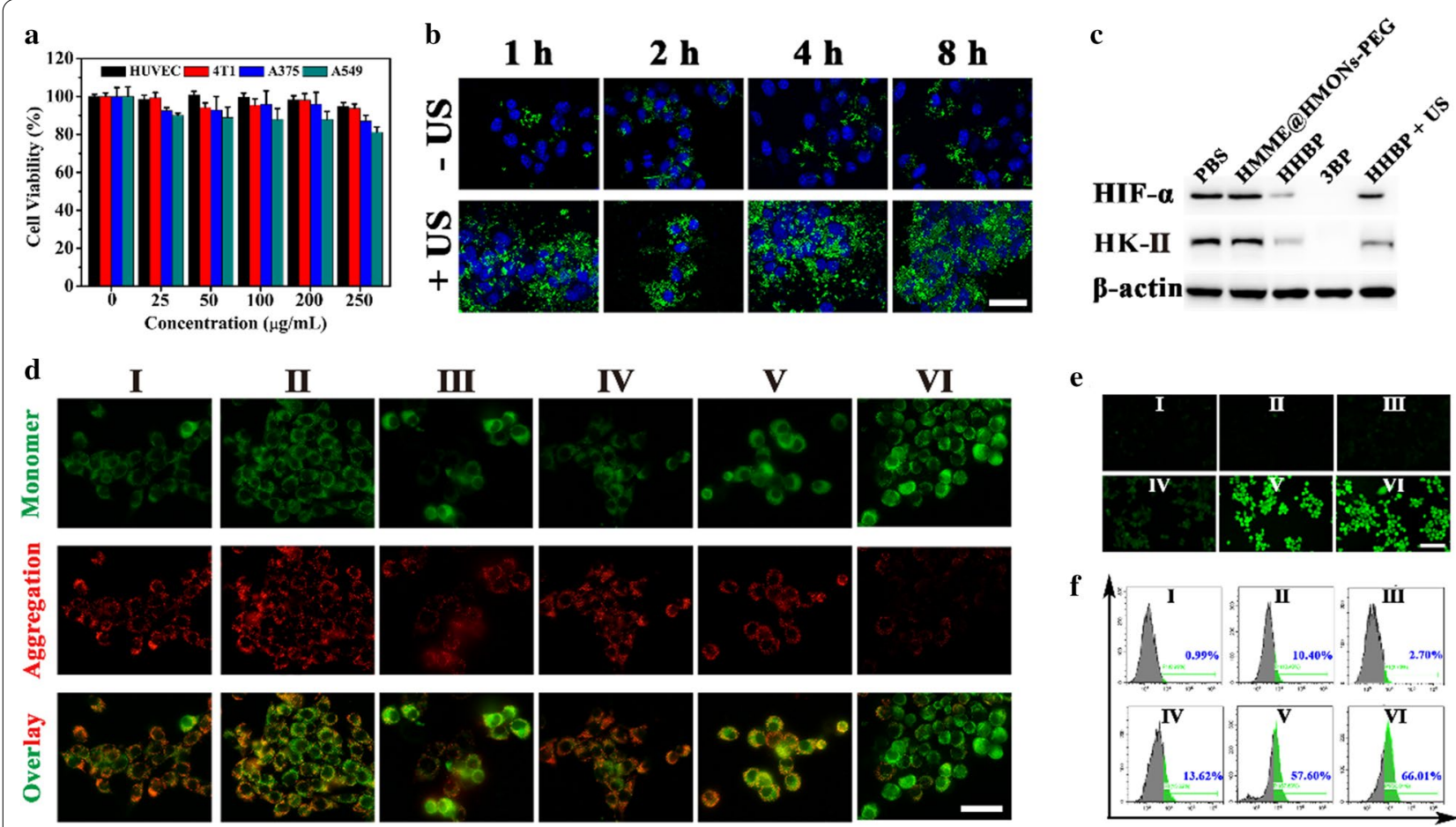

e

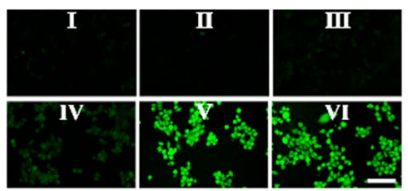
incubation with HMONs-PEG nanoparticles (concentrations of 25, 50, 100, 200, and $250 \mu \mathrm{g} / \mathrm{mL}$ ) for $24 \mathrm{~h}$. $\mathbf{b}$ CLSM images of 4 T1 cells incubated with FITC-conjugated HHBP for 1, 2, 4, and $8 \mathrm{~h}$ without or with US irradiation. Scale bar $=25 \mu \mathrm{m}$. c Western blotting results of HIF-1a and HK-II expression in $4 \mathrm{~T} 1$ cells after different treatments. Fluorescence images of 4T1 cells after different treatments and staining with $\mathbf{d} \mathrm{JC}-1$ dye and $\mathbf{e}$ an intracellular ROS probe, DCFH-DA. Scale bar $=50$ and $100 \mu \mathrm{m}$. f Flow cytometry data showing intracellular total ROS after different treatments. In panels $\mathbf{d}, \mathbf{e}$, and f treatment groups are denoted as: (I) PBS, (II) HMME@HMONs-PEG, (III) HHBP, (IV) PBS + US, (V) HMME@HMONs-PEG + US and (VI) HHBP + US

cellular uptake efficiency of HHBP in the cells was investigated. Confocal laser scanning microscopy (CLSM) observations indicated a time-dependent endocytosis process, as evidenced by the increased FITC signals at extended co-incubation durations $(1,2,4$, and $8 \mathrm{~h}$ ) of HHBP with $4 \mathrm{~T} 1$ cancer cells (Fig. 3b). More excitingly, after further irradiation with the US, a stronger green fluorescence was detected in 4T1 cells in the presence of HHBP. This phenomenon mainly due to the local cavitation effect induced minor disruptions to cell membrane for improving the cell uptake of nanoparticles [10]. In addition, flow cytometry analyses were also performed to determine the fluorescence intensity of FITC (Additional file 1: Fig. S10). The cells treated with HHBP exhibited a high uptake efficiency with increasing incubation time and the US stimulation could enhance the cellular uptake of nanoparticles which are consistent with the CLSM observation.

As an excellent respiration inhibitor, 3BP is expected to be a hypoxia ameliorator for reducing $\mathrm{O}_{2}$ consumption, which is the essential condition for amplifying SDT [49]. To verify this behavior, the expression of HK-II, which involved in the first stage of cellular respiration, was evaluated in the cells after treatment with different formulations by Western blotting (Fig. 3c). Notably, negligible changes in the HK-II and HIF- $1 \alpha$ levels were detected in $4 \mathrm{~T} 1$ cells after incubation with HMME@HMONs-PEG, while HHBP with or without US irradiation could markedly reduce the protein expression levels (Additional file 1: Fig. S11). Arguably, the introduction of 3BP played a vital role in the downregulation of HK-II and HIF-1 $\alpha$. These results prove that the inhibition of HK-II used by such an HHBP exposure could inhibit cellular respiration, which could cause changes in the cellular oxygen consumption pattern. Furthermore, the effect of HHBP under US irradiation on mitochondrial dysfunction was evaluated by measuring the variation in the mitochondrial membrane potential with the commercial JC-1 dye. We found that the $4 \mathrm{~T} 1$ cells treated with HHBP without or with US irradiation displayed strong green fluorescence (Fig. 3d), which is in marked contrast to the prominent red fluorescence observed after treatment with PBS or HMME@HMONs-PEG, thus indicating that the respiration was further suppressed. 


\section{In vitro augmented SDT efficacy of engineered HHBP} nanosonosensitizer at the cellular level

Inspired by the excellent $\mathrm{O}_{2}$-economization properties of HHBP nanosonosensitizers, the intracellular ROS generation and in vitro augmented SDT efficacy were further assessed. The intracellular ROS levels were visualized by a of DCFH-DA probe, which could be oxidized into green fluorescent, 2',7'-dichlorofuorescin (DCF) in the presence of ROS $[50,51]$. As revealed in Fig. 3e, negligible fluorescence was detected in the PBS, HMME@HMONs-PEG, and HHBP groups, while the cells treated with the latter two under the US irradiation displayed conspicuous green fluorescence, thus indicating a strong ROS generation. Further quantitative flow cytometric results summarized in Fig. 3f demonstrate that the cellular fluorescence intensity of HHBP + US treatment was significantly higher than that with other treatments. As a result,
HHBP could induce plentiful ROS production under US irradiation, displaying a significant potential for SDT against tumors. Encouraged by the prominent ROS generation performance of HHBP, in vitro augmented SDT effect was assessed on $4 \mathrm{~T} 1$ cells by the CCK- 8 assay. As expected, the viabilities of the cells after treatment with HMME@HMONs-PEG + US, 3BP-HMONs-PEG, 3BPHMONs-PEG + US, HHBP were decreased to $62.5 \%$, $53.8 \%, 62.2 \%$ and $44.9 \%$ at the HMME concentration of $38 \mu \mathrm{g} / \mathrm{mL}$, respectively (Fig. 4a). In contrast, much lower cell viabilities were detected after treatment with HHBP at the corresponding concentrations under US irradiation, suggesting an excellent synergistic effect of SDT and 3BP. Similar results were also revealed by the CalceinAM/PI stain assay (Fig. 4b). Moreover, the treatment of HHBP under US irradiation could also achieve an effective inhibition effect on diverse tumor cells including
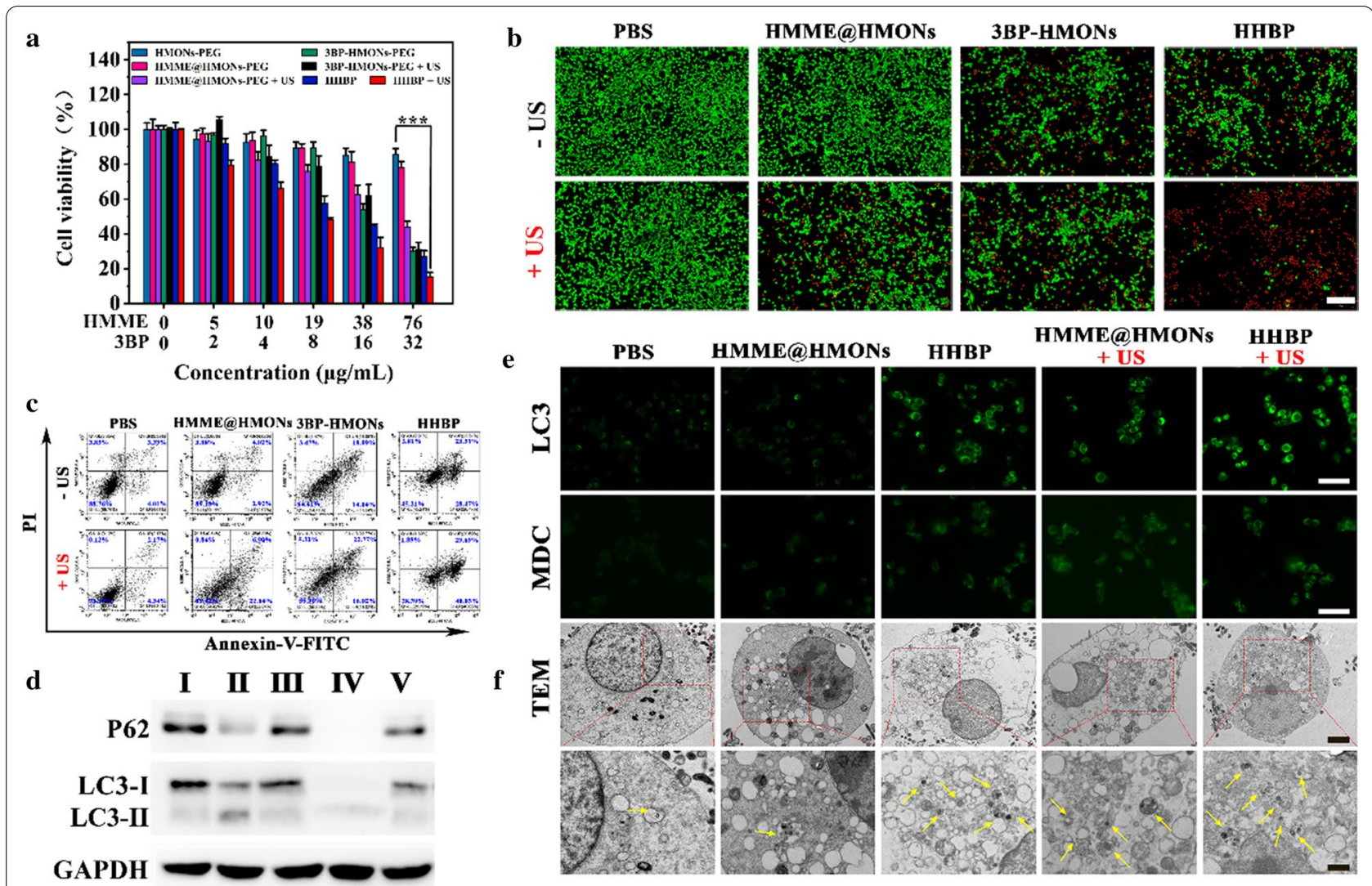

Fig. 4 Pro-death autophagy induced by HHBP promoted cancer cell apoptosis. a The relative viabilities of 4T1 cells after different treatments, quantified by CCK-8 assay $(n=6)$. ${ }^{* *} \mathrm{P}<0.001$. b Fluorescence images of 4 T1 cells stained by Calcein-AM (green) and PI (red) after treatment with PBS, HMME@HMONs, HHBP without or with US irradiation. Scale bar=200 $\mathrm{mm}$. c Flow cytometry analysis of annexin V-FITC/PI-stained 4T1 cells after treatment with PBS, HMME@HMONs or HHBP without or with US irradiation. d Western blotting of the p62, LC3-I, and LC3-II levels in 4T1 cells after different treatments. (I) PBS, (II) HHBP, (III) HMME@HMONs, (IV) HHBP + US, and (V) HMME@HMONs + US. e Representative immunofluorescence staining of LC3 punctate dots and MDC-stained fluorescent images after 4T1 cells were treated with HMME@HMONs or HHBP for 4 h with or without US irradiation. Scale bars=100 $\mathrm{mm}$. f TEM images showing the formation of autophagosomes after the 4T1 cells were treated with HMME@ HMONs or HHBP for $4 \mathrm{~h}$ with or without US irradiation. Scale bars $=2 \mu \mathrm{m}$. The yellow arrows in the magnification of TEM images represent the typical structures of autophagosomes. Scale bars $=1 \mu \mathrm{m}$ 
A375 and A549 cells with strong concentration-dependent cytotoxicity (Additional file 1: Fig. S12). Significantly, almost no green fluorescence was observed when the cells were treated with HHBP and US irradiation. In addition, the apoptosis effect induced by the synergistic effect was evaluated by the Annexin V-FITC/PI based Flow Cytometer assay (Fig. 4c). The apoptosis/necrosis rate of the cells treated with "HHBP + US" was $69.7 \%$, which was much higher compared with that of other treatments. Taken together, the HHBP nanosonosensitizer could reduce $\mathrm{O}_{2}$ consumption for enhanced SDT, thus promising improvement in the therapeutic outcomes of cancer treatment.

\section{Pro-death autophagy induced by HHBP promoted cancer cell apoptosis}

Notably, the in vitro therapeutic outcomes showed that HHBP treatment without US irradiation could also acquire moderate inhibitory effects. Therefore, we believe that 3BP implicates an essential role in inhibiting cell viabilities. In past studies, 3BP can suppress both mitochondrial respiration and glycolysis to result in starvation-induced autophagy [49]. To further verify the hypothesis that 3BP can induce pro-death autophagy, we evaluated the expression of the autophagy-related protein (LC3 and p62) in 4T1 cells receiving different treatments by Western blotting. Notably, the highest cellular autophagy levels were detected after treatment with HHBP plus US irradiation (Fig. 4d), which was proved by p62 inhibition as well as the LC3-II/LC3-I ratio elevation. Quantification results shown in Additional file 1: Fig. S13 further indicate that the LC3-II/LC3-I ratio increased by 98 -fold after the HHBP + US treatment when compared with that after the control treatment, while the expression of p62 reduced to $1.5 \%$. These observations demonstrated that the synergistic effect of 3BP and SDT could effectively induce autophagy in $4 \mathrm{~T} 1$ cells. Furthermore, immunofluorescence staining was performed to characterize the autophagy level by visualized monitoring of the LC3 punctate dots (Fig. 4e). Both HHBP alone and HMME@HMONs-PEG + US treatments exhibited green significant fluorescence signals, which can be attributed to the autophagy induced by $3 \mathrm{BP}$ and ROS, respectively. When combined with HHBP and extra US irradiation, the green fluorescence was significantly enhanced. The formation of acidic vesicular autophagosomes during the autophagy process was examined by performing the monodansylcadaverine (MDC) staining (Fig. 4e). The treatment of HHBP incubation plus US exposure resulted in extremely strong green fluorescence of acidic autophagosomes when compared to that of the cells treated with HHBP and HMME@HMONs-PEG + US. To observe the autophagosomes more intuitively, the
4T1 cells receiving different treatments were observed by TEM (Fig. 4f). An unequal quantity of autophagosomes was detected in all treatment groups except for the control group, thus indicating that 3BP or SDT-induced ROS could trigger cell autophagy. Meanwhile, HBBP incubated $4 \mathrm{~T} 1$ cells exposed by the US represented more autophagic vesicles than those in the other treatments. These results suggested that the synergistic effect can activate excessive autophagy, which in turn promote cell death. Concomitantly, the ATP and lactate level in 4T1 cells were investigated. As shown in Additional file 1: Fig. S14, HMME@HMONs-PEG treatment showed no obvious influence on the intracellular ATP level when compared to that in the control group. However, both 3BP and HHBP dramatically reduced the ATP to $17.8 \%$ and $20.2 \%$, respectively, suggesting that the depletion of ATP induced by $3 \mathrm{BP}$ could significantly elevate the autophagy level. The data revealed that the content of lactate in the treatment groups of 3BP-contained formulations were significantly lower than that in the control group (Additional file 1: Fig. S15), indicated that 3BP inhibited glycolysis and reduced the production of lactate. These results agree well with the therapeutic evaluations described earlier and offer reliable evidence that the improved anticancer activity of HHBP-mediated augmented SDT could be ascribed to the synergistic effect of apoptosis and autophagy.

\section{In vivo biodistribution of the HHBP nanosonosensitizer}

The in vitro outstanding properties of the nanosonosensitizer encouraged us to further assess the therapeutic performance on $4 \mathrm{~T} 1$ breast tumor xenografts in nude mice. For tracking the in vivo distribution and the tumor accumulation behavior of HHBP, a near-infrared dye, indocyanine green (ICG), was loaded to the HHBP through a simple mixing method. From the in vivo fluorescence images (Fig. 5a), the fluorescence signals at the tumor site were increased with increasing time, with the maximum signal detected at $12 \mathrm{~h}$ post-injection, which was also maintained at $16 \mathrm{~h}$, followed by a decline due to the degradation of ICG. Correspondingly, the tumor and the main organs of mice were excised at $24 \mathrm{~h}$ for ex vivo imaging. Obviously, the ICG-HHBP-treated mice showed strong ICG fluorescence activity at the tumor sites (Additional file 1: Fig. S16). Moreover, a strong fluorescence signal was detected in the liver, which may be attributed to the specific uptake of the mononuclear phagocyte system [52]. These results suggest the favorable tumor accumulation performance of HHBP. Furthermore, the blood circulation profile of the HHBP was evaluated by determining the concentration of Si element in blood collected from the mice at different time points. As shown in Additional file 1: Fig. S17, the pharmacokinetics 


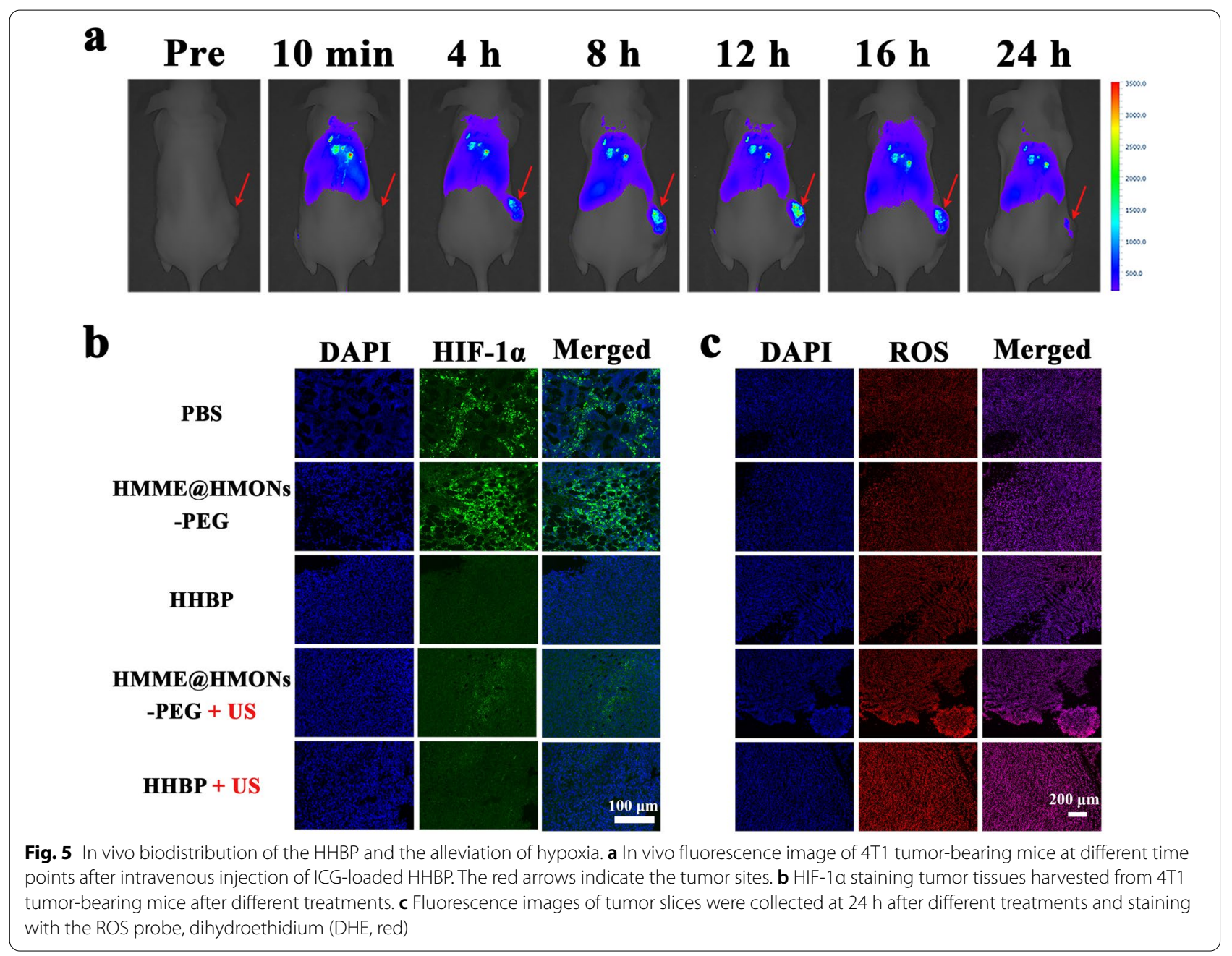

curve of HHBP followed a classical two-compartment model with a relatively long blood half-life $\left(\mathrm{t}_{1 / 2}=2.09 \pm\right.$ $0.37 \mathrm{~h}$ ). These results suggested that the obtained nanosonosensitizer features preferable blood circulation stability and has substantial potential for future clinical transformation.

\section{In vivo alleviation of hypoxia}

Based on the above-mentioned in vitro findings, HHBP was expected to be a hypoxia modulator for reducing $\mathrm{O}_{2}$ consumption, which is deemed beneficial to overcome tumor hypoxia and improve the SDT efficiency. Before testing the antitumor efficacy, the ability of HHBP to ameliorate tumor hypoxia was evaluated by HIF- $1 \alpha$ immunofluorescence staining assay. As observed under the fluorescence microscopy (Fig. 5b), extensive green fluorescence was detected in the PBS and HMME@ HMONs-PEG groups, thus confirming the overexpression of HIF-1 $\alpha$ under hypoxia. In contrast, the indicators of HIF-1 $\alpha$ were downregulated after treatment with
HHBP with or without US irradiation, which indicated that 3BP could effectively relieve tumor hypoxia.

\section{In vivo antitumor efficacy enabled by HHBP nanosonosensitizer}

To investigate whether the HHBP-mediated augmented SDT strategy was applied to the in vivo experiments, the therapeutic performance of HHBP against the 4T1 tumor-bearing mice model was performed. Briefly, the mice were randomly divided into 6 groups $(n=5)$, as follows: (I) PBS, (II) HMME@HMONs-PEG, (III) HMME@HMONs-PEG + US, (IV) HHBP, and (V) $\mathrm{HHBP}+\mathrm{US}$. The injected dosage was set to $8 \mathrm{mg} / \mathrm{kg}$ HMME and $3.4 \mathrm{mg} / \mathrm{kg} 3 \mathrm{BP}(100 \mu \mathrm{L})$. The tumor sites of the mice in Group III and V were exposed to US (1.5 W/ $\mathrm{cm}^{2}, 1.0 \mathrm{MHz}, 50 \%$ duty circle) for $5 \mathrm{~min}$ and twice as much US exposure on days 1 and 4, respectively. To examine the ROS level at the tumor sites, tumors from different treatment groups were collected after US irradiation for dihydroethidium staining (Fig. 5c). It was 
observed that both HMME@HMONs-PEG and HHBP could improve the ROS levels under US exposure, and both showed evidently higher levels relative to that with other treatments. More specifically, a higher ROS level could be achieved by HHBP + US treatment, which validated that $3 \mathrm{BP}$ can relieve tumor hypoxia and cause tremendous ROS generation through the amplification of the HMME-initiated sonodynamic effect. Following these treatments, the tumor volumes (Fig. 6a) and body weights (Fig. 6b) of the experimental mice were monitored over 2 weeks. We observed rapid tumor growth in the PBS and HMME@HMONs-PEG treated groups, but moderate tumor growth (inhibition rate: $40 \%$ and $28.5 \%$, respectively) in the HHBP and HMME@HMONs-PEG plus US treatment groups. More excitingly, the tumors on the mice treated with HHBP and US exposure were found to be most suppressed (inhibition rate: 89.1\%). The corresponding images of representative tumors on day 14 in different treatment groups further confirmed that the HHBP + US group realized an intelligent antitumor effect (Fig. 6c). In addition, there was a negligible body-weight loss in mice in all groups during the treatment (Fig. 6b), demonstrating the low adverse effects of all formulations in vivo. The abovementioned desirable therapeutic efficacy may be benefited from the excellent synergistic therapeutic effect, including the 3BP-mediated hypoxia modulation, HMME-based augmented SDT and 3BP-induced excessive activation of autophagy.

To further reveal the performance mechanism of HHBP, the apoptosis and autophagy levels of the collected tumors from all groups were evaluated. Compared to that with other treatments, we detected the most significant large-area histological damaged regions from the $\mathrm{H} \& \mathrm{E}$ staining, the most significant inhibition of cell proliferation from Ki-67 staining, and the highest evident apoptosis of tumor cells from TUNEL staining in the "HHBP + US" treatment group (Fig. 6d). These results are consistent with the foregoing results of in vivo anticancer experiments. Subsequently, we also examined the autophagy levels in tumor sections after different treatments by immunofluorescence imaging staining of LC3 (Fig. 6e). We found that both HMME@HMONs-PEG + US and HHBP alone could improve autophagy levels, according to the green fluorescence of LC3 puncta displayed in the immunofluorescence images. Consistent with the in vitro data, the HHBP group demonstrated the strongest green

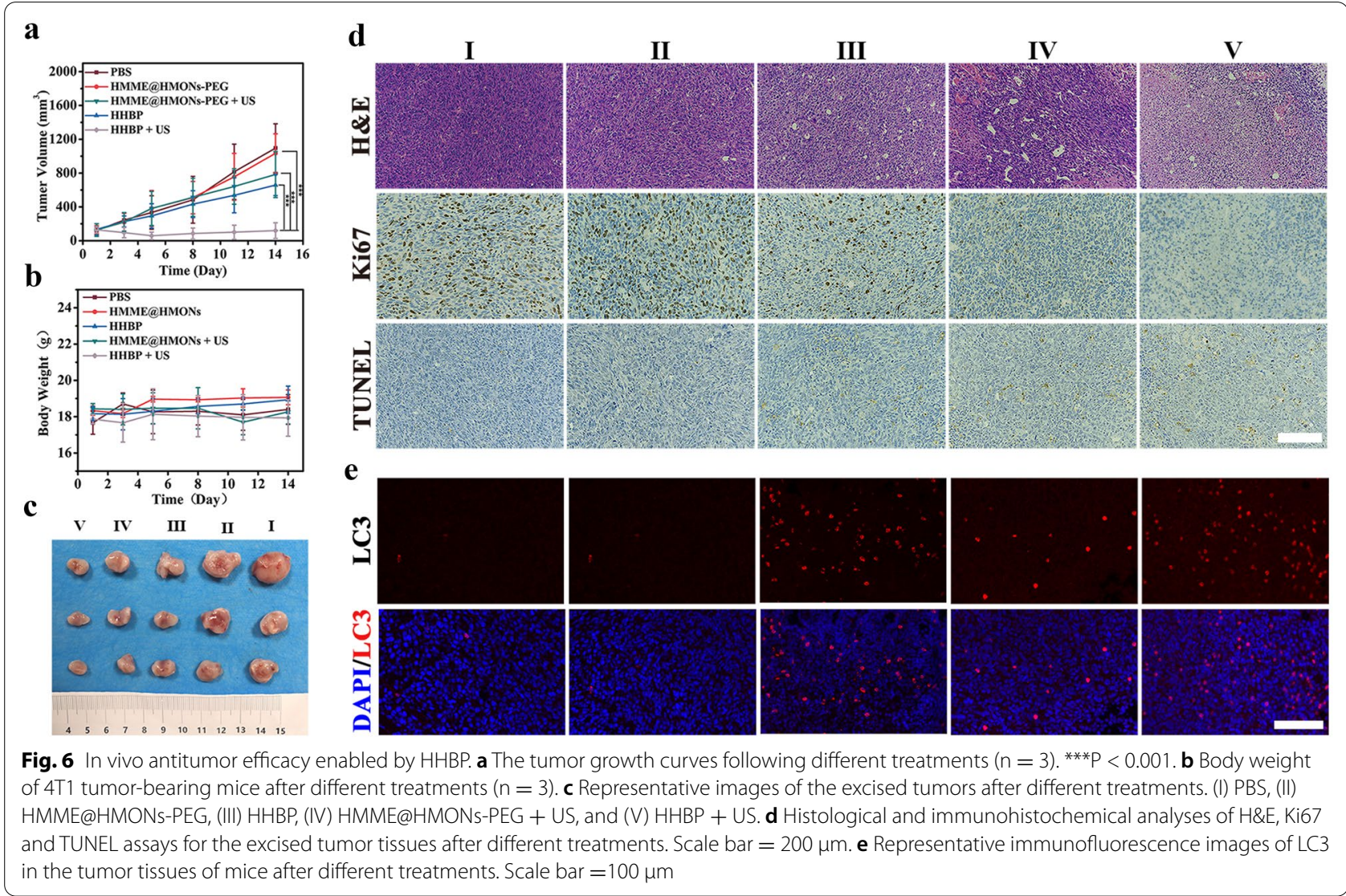


fluorescence after US irradiation, which represents an excessive activation of autophagy by 3BP and SDT.

Finally, in terms of the safety assessment of HHBP on mice, the H\&E sections of the collected major organs did not show any obvious inflammation after injection with HHBP for 3, 7, and 14 days (Additional file 1: Fig. S18), indicating the favorable biocompatibility of HHBP. Concomitantly, the blood sample was collected from healthy female Balb/c mice at the different time points postinjection of HHBP for blood biochemistry and blood routine examinations. No obvious changes were detected concerning the blood routine indexes and liver/kidney function associated biomarker induced by HHBP relative to those in the control group (Additional file 1: Fig. S19), which hints at ignorable systemic toxicity. These results indicated that the fabricated HHBP may augment SDT efficacy with only relatively low systematic toxicity under in vivo conditions.

\section{Conclusions}

In summary, we have proposed an $\mathrm{O}_{2}$-economizer strategy to reverse the tumor hypoxia for improving the therapeutic efficacy of SDT, as a substitute for the current mainstream hypoxia-regulation precept of elevating intratumoral $\mathrm{O}_{2}$ level. To validate the concept, a biodegradable reduced expenditure bioreactor (HHBP) was constructed through the integration of 3BP and HMME into biodegradable HMONs, which respond to TME and subsequently augments SDT efficacy. The 3BP inhibits cell respiration through downregulation of HK-II expression, which in turn decreases the $\mathrm{O}_{2}$ consumption and spares more $\mathrm{O}_{2}$, thereby facilitating the SDT by HMME against hypoxic tumors. Importantly, the enhanced ROS generation and 3BP can transform the actor of autophagy from self-protection to pro-death, which is indicated by the increase in the LC3 level and the inhibition of p62 expression. Consequently, HHBP nanosonosensitizers offer better efficient antitumor performance relative to that of the traditional SDT sonosensitizer, which has been systematically verified both in vitro and in vivo. This $\mathrm{O}_{2}$-economizer concept not only offers a general strategy to alleviate the tumor hypoxia in SDT but also paves a new way for manipulating autophagic processes to enhance the efficacy of ROS-mediated cancer therapy.

\section{Supplementary Information}

The online version contains supplementary material available at https://doi. org/10.1186/s12951-021-01166-y.

Additional file 1: Fig. S1.TEM images of $\mathrm{SiO}_{2} @ M O N s$ nanoparticles. Fig. S2. EDS spectrum of the prepared HMONs. Fig. S3. ${ }^{13} \mathrm{C}$ and (b) ${ }^{29} \mathrm{Si} \mathrm{NMR}$ spectra of HMONs. Fig. S4. FT-IR spectra of 3BP, HMME, and different HMONs-based nanoparticles. Fig. S5. $\mathrm{N}_{2}$ adsorption-desorption isotherm and pore-size distribution of HMONs, HMONs-NH 2 , HMME@HMONs-PEG, and HHBP nanoparticles. Fig. S6. zeta potentials of HMONs, HMONs-NH ${ }_{2}$ HMME@HMONs-PEG, and HHBP aqueous solution. Fig. S7. HMME loading capacities on HMONs-3BP-PEG NPs (w/w\%) with different HMONs-3BP/ HMME feeding ratios. Fig S8. Stability of HHBP in different solutions. Fig. S9. TEM images of HHBP dispersed in SBF at varied GSH concentrations $(0,5$, and $10 \mathrm{mM})$ for 1,5 , and 7 days. Fig. S10. Flow cytometry analyses of 4T1 cells incubated with FITC-conjugated HHBP. Fig. S11. Quantitative determination of the relative HIF-1 $a$ and HK-II expression from Western blotting results. Fig. S12. Relative viabilities of A549 and A375 cells after different treatments. Fig. S13. Quantitative analysis of LC3-II/LC3-I and relative p62 expression after different treatments from Western blotting results. Fig. S14. ATP levels in 4T1 cells after different treatments. Fig. S15. Intracellular lactic acid content of $4 \mathrm{~T} 1$ cells after incubation with different nanoparticles. Fig. S16. Ex vivo fluorescence imaging of the tumor and the major organs collected from the mice at $24 \mathrm{~h}$ post-injection. Fig. S17. Blood circulation lifetime of HHBP after intravenous injection into mice. Fig. S18. H\&E-stained histological images of major organs from mice treated with PBS versus HHBP. Fig. S19. Blood biochemistry and hematology data of Balb/c mice treated with HHBP at different time point after i.v. injection,

\section{Authors' contributions}

WZ: conceptualization, investigation, data curation, methodology, formal analysis, validation, writing original draft. JH: investigation, data curation, methodology, resources, software. JW: resources, supervision, writing review \& editing, project administration, funding acquisition. XC: writing review \& editing. BH: methodology, formal analysis, investigation. ZW: super-vision, resources. YZ: conceptualization, funding acquisition, writing review \& editing, supervision, project administration. All authors read and approved the final manuscript.

\section{Funding}

We greatly acknowledge the financial support from the NSFC Key Projects of International Cooperation and Exchanges (Grant No. 81720108023), National Natural Science Foundation of China (Grant No. 82001943), Translational Medicine National Science and Technology Infrastructure (Shanghai) open project fund (Grant No. TMSK-2020-004), the open project of State Key Laboratory of Oncogenes and Related Genes (Grant No. KF2104-93\#), and China Postdoctoral Science Foundation (2020M681331, 2021T140458).

\section{Availability of data and materials}

The datasets and materials used in the study are available from the corresponding author.

\section{Declarations}

\section{Ethics approval and consent to participate}

All the animal procedures were performed under the protocol approved by the Institutional Animal Care and Use Committee of Shanghai Jiao Tong University Medical College. All the animal experimental operations were in compliance with the National Guidelines for Animal Protection.

\section{Consent for publication}

All authors have approved the manuscript be submitted.

\section{Competing interests}

The authors declare no competing interests.

\section{Author details}

${ }^{1}$ Department of Ultrasound in Medicine, Shanghai Institute of Ultrasound in Medicine, Shanghai Jiao Tong University Affiliated Sixth People's Hospital, Shanghai 200233, People's Republic of China. ${ }^{2}$ Chongqing Key Laboratory of Ultrasound Molecular Imaging, Ultrasound Department of the Second Affiliated Hospital of Chongqing Medical University, Chongqing 400010, People's Republic of China. ${ }^{3}$ State Key Laboratory of Oncogenes and Related Genes, School of Medicine, Shanghai Jiao Tong University, Shanghai 200233, People's Republic of China. 
Received: 28 September 2021 Accepted: 28 November 2021 Published: 13 December 2021

\section{References}

1. Mitragotri S. Healing sound: the use of ultrasound in drug delivery and other therapeutic applications. Nat Rev Drug Discov. 2005;4:255.

2. Qian X, Zheng Y, Chen Y. Micro/nanoparticle-augmented sonodynamic therapy (SDT): breaking the depth shallow of photoactivation. Adv Mater. 2016;28:8097-129.

3. Zou MZ, Liu WL, Chen HS, Bai XF, Gao F, Ye JJ, et al. Advances in nanomaterials for treatment of hypoxic tumor. Natl Sci Rev. 2021;8:17.

4. Chen H, Zhou X, Gao Y, Zheng B, Tang F, Huang J. Recent progress in development of new sonosensitizers for sonodynamic cancer therapy. Drug Discov Today. 2014;19:502-9.

5. Rwei AY, Paris JL, Wang B, Wang W, Axon CD, Vallet-Regi M, et al. Ultrasound-triggered local anaesthesia. Nat Biomed Eng. 2017; 1: 644-53.

6. Geng P, Yu N, Zhang J, Jin Z, Wen M, Jiang Q, et al. One responsive stone, three birds: $M n$ (iii)-hemoporfin frameworks with glutathione-enhanced degradation, MRI, and sonodynamic therapy. Adv Healthcare Mater. 2020; 10: 2001463.

7. Zhu P, Chen Y, Shi J. Nanoenzyme-augmented cancer sonodynamic therapy by catalytic tumor oxygenation. ACS Nano. 2018; 12: 3780-95.

8. Jia Q, Ge J, Liu W, Zheng X, Chen S, Wen Y, et al. A magnetofluorescent carbon dot assembly as an acidic $\mathrm{H}_{2} \mathrm{O}_{2}$-driven oxygenerator to regulate tumor hypoxia for simultaneous bimodal imaging and enhanced photodynamic therapy. Adv Mater. 2018; 30: 1706090.

9. Xu H, Yu N, Zhang J, Wang Z, Geng P, Wen M, et al. Biocompatible Fehematoporphyrin coordination nanoplatforms with efficient sonodynamic-chemo effects on deep-seated tumors. Biomaterials. 2020; 257: 120239.

10. Tang H, Zheng Y, Chen Y. Materials chemistry of nanoultrasonic biomedicine. Adv Mater. 2017;29:1604105.

11. Xu T, Zhao S, Lin C, Zheng X, Lan M. Recent advances in nanomaterials for sonodynamic therapy. Nano Rese. 2020; 13: 2898-908.

12. Ding $B$, Zheng $P$, Jiang $F$, Zhao $Y$, Wang $M$, Chang $M$, et al. MnOx nanospikes as nanoadjuvants and immunogenic cell death drugs with enhanced antitumor immunity and antimetastatic effect. Angew Chem Int Ed Engl. 2020;59:1-5.

13. Ma Z, Jia X, Bai J, Ruan Y, Wang C, Li J, et al. $\mathrm{MnO}_{2}$ gatekeeper: An intelligent and $\mathrm{O}_{2}$-evolving shell for preventing premature release of high cargo payload core, overcoming tumor hypoxia, and acidic $\mathrm{H}_{2} \mathrm{O}_{2}$-sensitive MRI. Adv Funct Mater. 2017;27:1604258.

14. Chen Q, Feng L, Liu J, Zhu W, Dong Z, Wu Y, et al. Intelligent albumin$\mathrm{MnO}_{2}$ nanoparticles as $\mathrm{pH}-/ \mathrm{H}_{2} \mathrm{O}_{2}$-responsive dissociable nanocarriers to modulate tumor hypoxia for effective combination therapy. Adv Mater. 2016; 28: 7129-36.

15. Zhao J, Cui W. Functional electrospun fibers for local therapy of cancer. Adv Fiber Mater. 2020; 2: 229-45

16. Gao $\mathrm{S}$, Jin Y, Ge K, Li Z, Liu H, Dai X, et al. Self-supply of $\mathrm{O}_{2}$ and $\mathrm{H}_{2} \mathrm{O}_{2}$ by a nanocatalytic medicine to enhance combined chemo/chemodynamic therapy. Adv Sci. 2019; 6: 1902137.

17. Wang D, Wu H, Phua SZF, Yang G, Qi Lim W, Gu L, et al. Self-assembled single-atom nanozyme for enhanced photodynamic therapy treatment of tumor. Nat Commun. 2020; 11: 357.

18. Wei C, Liu Y, Zhu X, Chen X, Zhou Y, Yuan G, et al. Iridium/ruthenium nanozyme reactors with cascade catalytic ability for synergistic oxidation therapy and starvation therapy in the treatment of breast cancer. Biomaterials. 2020; 238: 119848.

19. Wu J, Wang X, Wang Q, Lou Z, Li S, Zhu Y, et al. Nanomaterials with enzyme-like characteristics (nanozymes): next-generation artificial enzymes (ii). Chem Soc Rev. 2019;48:1004-76.

20. Zhang X, Xi Z, Machuki JO, Luo J, Yang D, Li J, et al. Gold cube-in-cube based oxygen nanogenerator: a theranostic nanoplatform for modulating tumor microenvironment for precise chemo-phototherapy and multimodal imaging. ACS Nano. 2019;13:5306-25.

21. Sang Y, Cao F, Li W, Zhang L, You Y, Deng Q, et al. Bioinspired construction of a nanozyme-based $\mathrm{H}_{2} \mathrm{O}_{2}$ homeostasis disruptor for intensive chemodynamic therapy. J Am Chem Soc. 2020; 142: 5177-83.
22. Wang $T$, Zhang $H$, Liu H, Yuan Q, Ren F, Han Y, et al. Boosting $\mathrm{H}_{2} \mathrm{O}_{2}$-guided chemodynamic therapy of cancer by enhancing reaction kinetics through versatile biomimetic fenton nanocatalysts and the second near-infrared light irradiation. Adv Funct Mater 2019;30:1906128.

23. Wu B, Sun Z, Wu J, Ruan J, Zhao P, Liu K, et al. Nanoparticle-stabilized oxygen microcapsules prepared by interfacial polymerization for enhanced oxygen delivery. Angew Chem Int Ed Engl. 2021;60:1-7.

24. Yu W, Liu T, Zhang M, Wang Z, Ye J, Li CX, et al. O, economizer for inhibiting cell respiration to combat the hypoxia obstacle in tumor treatments. ACS Nano. 2019; 13: 1784-94.

25. Zhao LP, Zheng RR, Chen HQ, Liu LS, Zhao XY, Liu HH, et al. Self-delivery nanomedicine for $\mathrm{O}_{2}$-economized photodynamic tumor therapy. Nano Lett. 2020; 20: 2062-71.

26. Song X, Feng L, Liang C, Gao M, Song G, Liu Z. Liposomes co-loaded with metformin and chlorin e6 modulate tumor hypoxia during enhanced photodynamic therapy. Nano Res. 2017;10:1200-12.

27. Zhu J, Yu W, Liu B, Wang Y, Shao J, Wang J, et al. Escin induces caspasedependent apoptosis and autophagy through the ROS/p38 mapk signalling pathway in human osteosarcoma cells in vitro and in vivo. Cell Death Dis. 2017; 8: e3113.

28. Scherz-Shouval R, Elazar Z. Regulation of autophagy by ROS: Physiology and pathology. Trends Biochem Sci. 2011; 36: 30-8.

29. Sharma M, Pandey R, Saluja D. ROS is the major player in regulating altered autophagy and lifespan in sin-3 mutants of c. Elegans. Autophagy. 2018; 14: 1239-55.

30. Patricia Boya FR, Patrice Codogno. Emerging regulation and functions of autophagy. Nat Cell Biol. 2013; 15: 713-20.

31. Rubinsztein DC, Gestwicki JE, Murphy LO, Klionsky DJ. Potential therapeutic applications of autophagy. Nat Rev Drug Discov. 2007; 6: 304-12.

32. Galluzzi L, Pietrocola F, Levine B, Kroemer G. Metabolic control of autophagy. Cell. 2014; 159: 1263-76.

33. Wang T, Hu J, Luo H, Li H, Zhou J, Zhou L, et al. Photosensitizer and autophagy promoter coloaded ROS-responsive dendrimer-assembled carrier for synergistic enhancement of tumor growth suppression. Small. 2018; 14: 1802337.

34. Wang Y, Lin Y, Wang J, Qiao S, Liu Y, Dong W, et al. In situ manipulation of dendritic cells by an autophagy-regulative nanoactivator enables effective cancer immunotherapy. ACS Nano. 2019; 13: 7568-77.

35. Feng X, Wang P, Liu Q, Zhang T, Mai B, Wang X. Glycolytic inhibitors 2-deoxyglucose and 3-bromopyruvate synergize with photodynamic therapy respectively to inhibit cell migration. J Bioenerg Biomembr. 2015; 47: 189-97.

36. Ko YH, Smith BL, Wang Y, Pomper MG, Rini DA, Torbenson MS, et al. Advanced cancers: Eradication in all cases using 3-bromopyruvate therapy to deplete atp. Biochem Biophys Res Commun. 2004; 324: 269-75.

37. Wu J, Niu S, Bremner DH, Nie W, Fu Z, Li D, et al. A tumor microenvironment-responsive biodegradable mesoporous nanosystem for antiinflammation and cancer theranostics. Adv Healthcare Mater. 2019; 9: 1901307.

38. Wu J, Williams GR, Niu S, Gao F, Tang R, Zhu LM. A multifunctional biodegradable nanocomposite for cancer theranostics. Adv Sci. 2019;6:1802001.

39. Huang $P$, Chen $Y$, Lin $H$, Yu L, Zhang L, Wang L, et al. Molecularly organic/ inorganic hybrid hollow mesoporous organosilica nanocapsules with tumor-specific biodegradability and enhanced chemotherapeutic functionality. Biomaterials. 2017; 125: 23-37.

40. Li L, Yang Z, Fan W, He L, Cui C, Zou J, et al. In situ polymerized hollow mesoporous organosilica biocatalysis nanoreactor for enhancing ROSmediated anticancer therapy. Adv Funct Mater. 2019; 30: 1907716.

41. Du X, Kleitz F, Li X, Huang H, Zhang X, Qiao S-Z. Disulfide-bridged organosilica frameworks: Designed, synthesis, redox-triggered biodegradation, and nanobiomedical applications. Adv Funct Mater. 2018:28:1707325.

42. Lu N, Fan W, Yi X, Wang S, Wang Z, Tian R, et al. Biodegradable hollow mesoporous organosilica nanotheranostics for mild hyperthermiainduced bubble-enhanced oxygen-sensitized radiotherapy. ACS Nano. 2018; 12: 1580-91.

43. Wang M, Tan Y, Li D, Xu G, Yin D, Xiao Y, et al. Negative isolation of circulating tumor cells using a microfluidic platform integrated with streptavidinfunctionalized plga nanofibers. Adv Fiber Mater. 2021; 3: 192-202. 
44. Chen Y, Meng Q, Wu M, Wang S, Xu P, Chen H, et al. Hollow mesoporous organosilica nanoparticles: a generic intelligent framework-hybridization approach for biomedicine. J Am Chem Soc. 2014;136:16326-34.

45. Gong F, Yang N, Wang X, Zhao Q, Chen Q, Liu Z, et al. Tumor microenvironment-responsive intelligent nanoplatforms for cancer theranostics. Nano Today. 2020;32:100851.

46. Liu Y, Wu F, Ding Y, Zhu B, Su Y, Zhu X. Preparation and characterization of paclitaxel/chitosan nanosuspensions for drug delivery system and cytotoxicity evaluation in vitro. Adv Fiber Mater. 2019; 1: 152-62.

47. Li D, Lin L, Fan Y, Liu L, Shen M, Wu R, et al. Ultrasound-enhanced fluorescence imaging and chemotherapy of multidrug-resistant tumors using multifunctional dendrimer/carbon dot nanohybrids. Bioact Mater. 2021; 6: 729-39.

48. Lin X, Liu S, Zhang X, Zhu R, Chen S, Chen X, et al. An ultrasound activated vesicle of janus Au-MnO nanoparticles for promoted tumor penetration and sono-chemodynamic therapy of orthotopic liver cancer. Angew Chem Int Ed Engl. 2020;132: 1699.

49. Deng Y, Song P, Chen X, Huang Y, Hong L, Jin Q, et al. 3-bromopyruvateconjugated nanoplatform-induced pro-death autophagy for enhanced photodynamic therapy against hypoxic tumor. ACS Nano. 2020; 14: 9711-27.

50. Gong F, Cheng L, Yang N, Betzer O, Feng L, Zhou Q, et al. Ultrasmall oxygen-deficient bimetallic oxide mnwox nanoparticles for depletion of endogenous GSH and enhanced sonodynamic cancer therapy. Adv Mater. 2019; 31: e1900730

51. Sun Y, Sun X, Li X, Li W, Li C, Zhou Y, et al. A versatile nanocomposite based on nanoceria for antibacterial enhancement and protection from apdt-aggravated inflammation via modulation of macrophage polarization. Biomaterials. 2021; 268: 120614

52. Du JZ, Li HJ, Wang J. Tumor-acidity-cleavable maleic acid amide (TACMAA): A powerful tool for designing smart nanoparticles to overcome delivery barriers in cancer nanomedicine. Acc Chem Res. 2018;51:2848.

\section{Publisher's Note}

Springer Nature remains neutral with regard to jurisdictional claims in published maps and institutional affiliations.

Ready to submit your research? Choose BMC and benefit from:

- fast, convenient online submission

- thorough peer review by experienced researchers in your field

- rapid publication on acceptance

- support for research data, including large and complex data types

- gold Open Access which fosters wider collaboration and increased citations

- maximum visibility for your research: over $100 \mathrm{M}$ website views per year

At BMC, research is always in progress.

Learn more biomedcentral.com/submissions 